\title{
Wächst die Gefahr von Pandemien?
}

\section{Reinhard Burger}

In den letzten drei Jahrzehnten identifizierte man im Schnitt etwa jährlich einen neuen Erreger oder einen neuartigen bekannten Erreger in einzelnen Regionen der Welt. Hierzu gehörten bakterielle und virale Erreger und auch das auslösende Agenz von Prionerkrankungen. Einzelne Erreger haben das Potential, eine Pandemie auszulösen. Unter einer Pandemie versteht man eine sich weltweit ausbreitende Epidemie. Das Auftreten derartiger Erreger und deren Ausbreitung werden von einer Reihe von Faktoren bestimmt, die sich entweder spontan in der Natur ergeben oder durch menschliches Handeln bedingt sind. Hierzu gehört eine Anpassung mikrobieller Erreger an den menschlichen Wirt und Wechsel in den Eigenschaften. Die Suszeptibilität der Bevölkerung oder Einzelner kann den Infektionserfolg beeinflussen. Klima und Wetter stellen Parameter dar, die eine leichtere Ausbreitung mit sich bringen können. Trockenheit oder aber Nässe können die Verbreitung von Vektoren (Nager oder Stechmücken) beeinflussen. Besiedlung zuvor unbewohnter Landstriche kann Kontakt zu zoonotischen Erregern mit sich bringen, d. h. Erregern, die vom Tier auf den Menschen übertragen werden können. Die demographische Entwicklung und menschliches Verhalten gehen einher zum Teil mit erhöhter Infektionswahrscheinlichkeit, z. B. Anfälligkeit der alternden Bevölkerung oder Promiskuität. Die Bildung von „MegaCities" führt zu unzureichender Hygiene und sanitären Infrastrukturen.

Die Dichte an Flugverbindungen führt zu einem raschen und intensiven Austausch von Menschen und Waren im weltweiten Maßstab. Änderungen der Lebensmitteltechnologien können bei bakterieller Kontamination über den globalen Handel zu einer raschen Verbreitung kontaminierter Waren führen. In vielen Bereichen der Erde sind Public Health-Systeme unzureichend. Armut und soziale Ungleichheit erschweren in vielen Gebieten selbst Grundversorgung und Infektionsprävention bzw. Behandlung. Kriege und Hungersnöte stellen ein zusätzliches Hindernis dar. Nicht zuletzt gibt es auch Beispiele fehlenden politischen Willens zur Abhilfe, z. B. HIV-Bekämpfung in Südafrika noch vor wenigen Jahren. Schließlich sind bioterroristische Anschläge zum Ausbringen von gefährlichen Erregern nicht auszuschließen.

Eine Pandemie kann auftreten, wenn ein neuer oder neuartiger Erreger auftritt, der auf eine Bevölkerung trifft, die nicht durch Immunität nach früheren Infektionen oder Impfungen geschützt ist und wenn dieser Erreger leicht von Mensch zu Mensch übertragbar ist und bei einem bestimmten Anteil der Bevölkerung eine Erkrankung auslöst. Derartige Erreger stellen für die Gesundheitsbehörden 
eine große Herausforderung dar. Es gilt den tatsächlichen Erreger zu identifizieren und seine Epidemiologie aufzuklären, einschließlich Übertragbarkeit und Suszeptibilität. Die Pathomechanismen und Virulenz müssen beurteilt werden und die Risikofaktoren für eine Infektion (z. B. Vektoren). Nach einer raschen Identifizierung des Erregers gilt es dessen Eigenschaften zu definieren und eine robuste Diagnostik zu etablieren mit belastbaren Screening-Systemen. Aus den Eigenschaften des Erregers ergeben sich Maßnahmen zur Prävention und Elimination. Diese Maßnahmen bedingen eine verlässliche Infrastruktur.

Maßnahmen zur Bekämpfung einer pandemischen Ausbreitung bedingen frühzeitige und abgestimmte, länderübergreifende Maßnahmen. Die ökonomischen Konsequenzen derartiger Epidemien oder Pandemien sind beträchtlich und rechtfertigen entsprechende Präventionsmaßnahmen. Die Maßnahmen gegen biologische Bedrohungen nach den Ereignissen vom 11. September 2001 verbesserten den Vorbereitungsstand für das Auftreten neuer Erreger deutlich.

In dem letzten Jahrzehnt stellen West Nil Virus, SARS und das neue pandemische Virus Influenza A H1N1 Beispiele dar für neue beziehungsweise neuartige Erreger, die das Potential haben zu einer Kontinenten- übergreifenden Ausbreitung und Pandemie. 